\title{
A Localization Algorithm of Multi-hop Three Dimensional AOA with Space-based Angle Transmission and Its Application
}

\author{
Qingzhang Chen, Yunfeng Ni, Xinghua Li, Rongjie Wu, Yanjing Lei \\ Department of Computer , Zhejiang University \\ of Technology,Hangzhou310023,China \\ qzchen@zjut.edu
}

\author{
Shuojin Fang \\ Zhejiang Vocational College of Commerce, \\ Hangzhou 310053, China \\ Anna_jin111@hotmail.com
}

\begin{abstract}
Wireless sensor node's localization is a funda-mental technology in Wireless Sensor Networks. There are only quite a few study on three-dimensional (3D) localization which is suffered in slow progress, actually, is one of the main difficulties in WSN localization. Based on the study of the existing two-dimensional positioning algorithm and the application of terrain modeling, localization algorithm for sensor nodes in (3D) condition has been focus on as well as the application of terrain model. Using the idea proposed by representative algorithm--APS multi-hop AOA (Angle of Arrival), this paper proposed a new algorithm named Multi-hop Three Dimensional AOA With Space-based Angle Trans-mission (MSAT3D AOA). Using this technology, target nodes can use information of anchor nodes which are more than one hop away form. This paper also combined MSAT3D AOA algorithm with Delaunay triangulation algorithm for terrain modeling.
\end{abstract}

Keywords-- three dimensional localization, wireless sensor network, angle transmission, $\mathrm{AOA}$, terrain modeling

\section{INTRODUCTION}

With the development of the economy and society, Wireless Sensor Network (WSN) has become the most influential technology of the century and will change human's way of life in the future as one of the four pillar industries of high-tech fields [1]. WSN is a self-organizing network, it consists of a large number of tiny sensor nodes with communication and computing capabilities, which are densely deployed in unattended monitoring region, and interconnected, processing and transmitting information through wireless communication. WSN is an integrated high and new technology of cross multi-disciplinary, high "integration" [2]. Most nodes are unknown in the network [3], the random distribution of nodes and the perceptive information depend on the location, making nodes localization exactly by themselves is an extremely important problem. With the development of the Internet of Things (IoT), add the ability of terrain modeling into sensor node, in order to support building terrain model of deployment environment, which will play a crucial role for WSN application. Whether military application, or the place where may prone to natural disasters or suffer natural disasters damage and the staff can't reach, if we can make use of node location information for terrain modeling, it will effectively help related work Smoothly. Therefore, the study of $3 \mathrm{D}$ localization and apply to terrain modeling is necessary and valuable.

\section{SENSOR LOCALIZATION RELATED WORKS}

Recently, researchers have proposed a number of 3D localization algorithm, such as Landscape-3D[4], T-3D[5], M-Based 3D Localization [6]. Common localization methods can be divided into two kind, they are distance based and distance independent. Usually [7], distance based localization technology is superior to distance independent in localization accuracy, though the former need additional hardware equipment, which will increase the cost and energy. Therefore, this paper will be based on AOA (Angle of arrival, the signal arrival angle) localization.

DV-Hop [8] which is proposed by D Niculescu and B Nath, is a distance independent multi-hop localization algorithm. 3D DV-Hop is the natural application of DV-Hop in 3D space. Though the algorithm is simple and easy to implement, its localization accuracy is unsatisfactory.

So far, scholars have proposed a variety of two dimensional localization algorithm according to different application, such as APS by D Niculescu and B Nath [9], SPA by Srdjan Capkum [10]. However, sensor nodes are often deployed in 3D space, if using two dimensional localization algorithm, there will be subject to the limita-tions of algorithm itself, and can't meet the requirements. Currently, there is only a little study in 3D localization, but the demand has become increasingly urgent. $3 \mathrm{D}$ localization is one of the important issues for IoT, and is one of core issues in WSN [11].

\section{A LOCALIZATION ALOGORITHM OF MULTI- HOP THREE DIMENSIONAL AOA WITH SPACE-BASED ANGLE TRANSMISSION}

Assuming that all nodes in the network have a spindle, representing self-positive direction, usually known as vertical of two receivers' connection [12]. Make use of the spindle estimating azimuth neighbor relatives to it, that is, the direction of neighbor to send data. Then we can use two beacons and their azimuths formed with the node to be located to get its location information. Similarly, if we assume that beacons are not only have the ability of measuring AOA on $x-y$ plane, but also have the ability to get signal arrival pith angle, then the unknown nodes can be located in 3D space by this new variable. This method is called simple 3D AOA, and on this basis, to improve node localization coverage, this paper proposed Multi-hop Three Dimensional AOA With Space-based Angle Transmission (MSAT 3D AOA). 


\section{A. The Principle of Angle Transmission}

AOA based multi-hop localization was first proposed by D Niculescu and B Nath to address Ad-Hoc network routing algorithm [13], its core technology is that nodes to be located have the ability to get information of beacons outside of its direct communication by other neighbors' angle transmission.

As shown in Figure 1, $L$ is beacon node, $A, B, C$ are all neighbor unknown nodes and have the ability to measure AOA. Assume that $B$ and $C$ have measured localization information with $L$. At the same time they measured each other's location information, and location information between $A$ and $B, C$. Hence, all the internal angles of $\triangle A B C$ and $\triangle L B C$ can be calculated and determined, and the shape of quadrilateral $A B C L$ and the angles formed by diagonals with edges can also be determined. So through these angle information, sine and other mathematical methods, $A$ can get angle formed by $L$ with its spindle. That is location information between $A$ and $L$ who is outside of its direct communication is determined.

As shown in Figure 2, A, B, $C$ are the unknown nodes with ability of measuring AOA, and adjacent each other, $L$ is a beacon node with omnidirectional antenna, but have no capacity of AOA, and $A$ is node to be located. In Figure 2, $A^{\prime}, B^{\prime}, C^{\prime}, L^{\prime}$ are $A, B, C, L$ projection positions on $x-y$ plane, respectively, and the relationship between these projection are as shown in Figure3-3. In Figure3-3, $\angle B_{1}, \angle B_{2}, \angle C_{1}, \angle C_{2}$ and $\angle B^{\prime} A^{\prime} C^{\prime}$ can be measured directly by angle measuring device. Moreover, we define $\angle A^{\prime}$ as $\angle B^{\prime} A^{\prime} C^{\prime}, \angle B^{\prime}$ as $\angle A^{\prime} B^{\prime} L^{\prime}$, $\angle C^{\prime}$ as $\angle A^{\prime} C^{\prime} L^{\prime}$.

Solving Formula (1) can get $\angle A_{1}$ and $\angle A_{2}$. Azimuth between $A^{\prime} L^{\prime}$ and absolute reference direction can be got by addition and subtraction of azimuth information about $\angle A_{1}$ or $\angle A_{2}$ or $A^{\prime} B^{\prime}$ or $A^{\prime} C^{\prime}$. Then $A$ gets the azimuth with $L$ who is outside of its direct communication. Since the location of these four nodes are different in the space, besides Figure 3, there are other five kinds of projection location on $x-y$ plane, we will not introduce here. When nodes are in different location relationships, their angles' relationships will be changed, and this also determines how to get azimuth information between $A$ and $L$ who is outside of its direct communication by measuring and calculated angles.

Figure 4 is the projection of node in Figure 2 on the plane of $A L L$. It can be proved by geometry of space that, the plane perpendicular to $x-y$ plane. In Figure 4, we define $B^{\prime \prime}$ and $C^{\prime \prime}$ as projection of $B$ and $C$ on plane of $A L L^{\prime}$, respectively.

The ability of nodes perception of 3D AOA is come from measured pitch angle which is relative to $x-y$ plane. However, only use these two AOA pitch angle can not determine their angle formed by projection on $A L L$ plane. Therefore, this paper uses relationship between projection on $x-y$ plane, to classify pitch angles projected on
$A L L$ ' plane. We take the relationship between $A, B$ and $C$ as an example, to explain how to classify and calculate angle between projected pitch angles on $A L L$ plane.

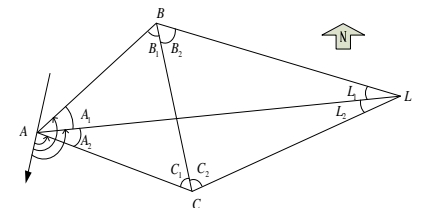
Schematic Diagram

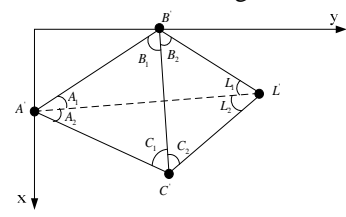

Fig 3.Node Projection $x$ - $y$ plane
Fig 1.Angle Transmission

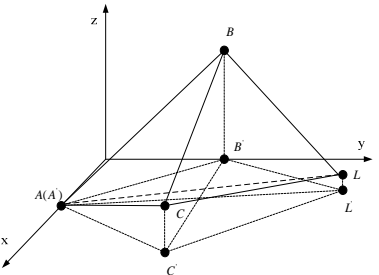

Fig 2.Three Dimensional Diagram

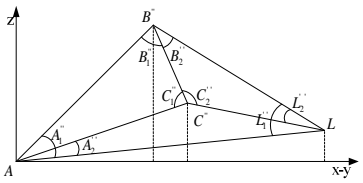

Fig 4.Node Projection on the Plane of $A L L$

$$
\begin{aligned}
& \int \frac{\sin \left(\pi-B^{\prime}-A_{1}\right)}{\sin A_{1}}=\frac{\sin C_{1} \cdot \sin \left(2 \pi-A^{\prime}-B_{1}-B_{2}-C_{1}-C_{2}\right)}{\sin C_{2} \cdot \sin A^{\prime}} \\
& \left\{\frac{\sin \left(\pi-C^{\prime}-A_{2}\right)}{\sin A_{2}}=\frac{\sin B_{1} \cdot \sin \left(2 \pi-A^{\prime}-B_{1}-B_{2}-C_{1}-C_{2}\right)}{\sin B_{2} \cdot \sin A^{\prime}}\right. \\
& \left\langle A=\left|\angle A B^{\prime \prime}-\angle A C^{\prime \prime}\right|\right. \\
& \angle B^{\prime \prime}=\pi-|\angle B " A+\angle B " L| \\
& \angle C^{\prime \prime}=\pi-\left|\angle C^{\prime \prime} A+\angle C^{\prime \prime} L\right| \\
& \angle B_{1}^{\prime \prime}=\pi-\left|\angle B^{\prime \prime} C+\angle B^{\prime \prime} A\right| \\
& \angle B_{2}^{\prime \prime}=\left|\angle B^{\prime \prime} L-\angle B^{\prime \prime} C\right| \\
& \angle C_{1}^{\prime \prime}=\left|\angle C B^{\prime \prime}-\angle C^{\prime \prime} A\right| \\
& \angle C_{2}^{\prime \prime}=\pi-\left|\angle C^{\prime \prime} B^{\prime \prime}-\angle C^{\prime \prime} L\right|
\end{aligned}
$$

Refer to Figure 3, $A^{\prime}, B^{\prime}$ and $C^{\prime}$ is the projection of $A, B$ and $C$ on $x-y$ plane. For $B^{\prime}$, plane azimuth formed by $A^{\prime}$ and itself is the direction of vector $\overrightarrow{B^{\prime} A^{\prime}}$, plane azimuth formed by $C^{\prime}$ and itself is the direction of vector $\overrightarrow{B^{\prime}}$. We require that, if there is an obtuse angle formed by any vector and vector $\overrightarrow{A L}$, then the vector represent the projection azimuth on $x-y$ plane, and the projection pitch angle of the projection azimuth on $A L L^{\prime}$ plane belongs to first kind pitch angle. Otherwise, if there is an acute angle or a right angle formed by them, then belongs to second kind. As a result, we can determine that, for $B, A$ and $C$ belongs to first kind and second kind, respectively.

Then we can calculate relationships between angles and pitch angles in Figure 4, as shown in Formula (2). Now, by this method, using $B$ and $C$, and relationship with $L, A$ can get 3D azimuth of $L$ relative to itself. Specifically, projection of $A, B, C, L$ on $A L L$ 'plane should be the same to projection on $x-y$ plane, there will be six relationships. Since are not projection nodes $A L L^{\prime}$ on plane, the length of $A L$ is the actual distance, and other projection on $A L L$ ' plane, before projected, their actual distance must be 
longer than projection length, cases that any projection longer than $A L$ will be not happen[17].

\section{B. Algorithm Description}

The core idea of MSAT3D AOA is based on two azimuths formed by two beacon nodes and node to be located, and two straight lines formed by azimuths with beacons, to determine node coordinates.[18] And on this basis, integrated angle transmission, through access to itself azimuth relative to beacon outside radio frequency, accomplish node localization using beacons outside of its direct communication.[19]

MSAT3D AOA has two parts, they are parameter configuration and actual operation. The relevant parameters needed in the localization process must be pre-written into all nodes, include: 1) hop-threshold, 2) manual setting or GPS obtaining beacons coordinates, 3) initializing un-knowns, and determine angle relationships formed by spindle direction and absolute reference direction through its own electronic compass, in order to calculating node coordinates in global coordinate. We assume that, under the direction of $x$ axis of the coordinates is north, under the direction of $Z$ axis is pointing to the opposite to center of the earth.[15]

According to the sequence of MSAT3D AOA operations, localization process can be summarized to the following five steps: First step is initializing node information in the network. Second step is localization for unknown nodes. Step three is judging HopMax. HopMax minus 1, which is stored in node, if HopMax is less than or equal to 0, then stop, and make unknown node into sleep mode, otherwise continue to step four.Step four is the information exchange of unknown nodes' beacons. Step five is analyzing "Neighbor Neighbor BeaconInfo". If all beacons have been dealing with, then return to step two. Step six is getting beacons information outside of direct communication. [16]

\section{Simulation and Analysis}

In this paper, algorithm simulation will be taken under MATLAB, and compare to DV-Hop localization algorithm. Assume that there are 100 nodes in $100 \mathrm{~m} \times 100 \mathrm{~m} \times 100 \mathrm{~m} 3 \mathrm{D}$ space. To make results more precisely, we use statistical methods, and test 50 times in the same network parameters experiment. For different algorithms of the same network parameter settings, we use the same data of nodes deployment. The main performance indicators: 1) Local-ization Error Ratio(LER), that is the average distance between estimated and real coordinates of to be located nodes, 2) Localization Cover Ratio (LCR), that is the percentage of to be located nodes in all unknown nodes, 3 ) Bad Node Proportion (BNP), that is the percentage of bad nodes in all to be located nodes, bad nodes mean localization error is greater than communications.

The experiment tests different performance of MSAT3D AOA under different HopMax in same communication ranges. Node communication range (Range) is set to $35 \mathrm{~m}$, the proportion of beacon is $5 \% \sim 30 \%$, we test the performance under the situation that we compared LER, LCR and BNP of this network under the situation that
HopMax is 3 and 5.

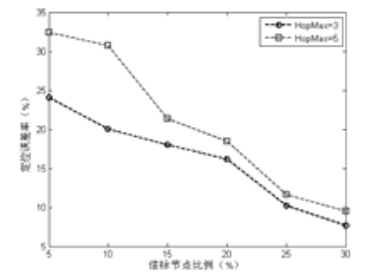

(a)

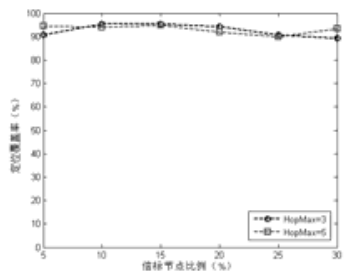

(b)

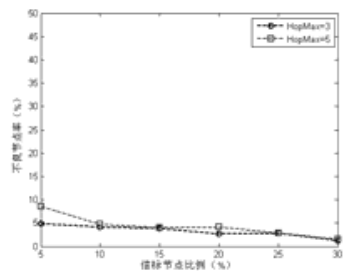

(c)

Fig 5. Simulation Results

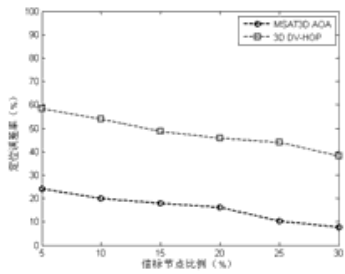

(a)

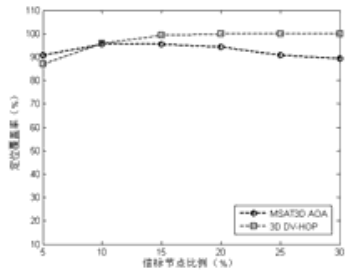

(b)

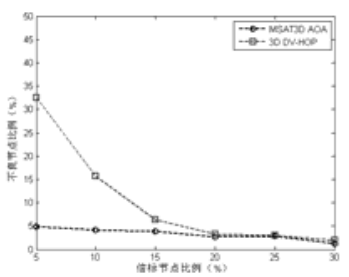

(c)

Fig 5. Simulation Results

In Figure 5, we can clearly find that MSAT3D AOA is really suitable to some specific applications, such as $3 \mathrm{D}$ WSN according to the three main performance indicators.

In Figure 6, compare with 3D DV-HOP according to the three main performance indicators we can also clearly find that MSAT3D AOA is much better than 3D DV-HOP.

\section{APPLICATION OF TERRAIN MODELING}

\section{A. Terrain Modeling Based on Delaunay Triangulation}

This paper uses Delaunay triangulation to construct triangulation in order to implement WSN based terrain modeling of deployment. Delaunay triangulation includes incremental insertion method, divide and conquer method, triangulation growth method three categories [14].

\section{Results Analysis and Comparison}

After localization, by making use of node(x, y) to form a point on the $\mathrm{x}-\mathrm{y}$ plane in 3D space, and using Delaunay incremental insertion method to construct triangular network, and then as basic for terrain modeling. After adding $\mathrm{z}$ coordinate to each vertex of triangles, each triangle 
will represent a patch in the space, and the graph consists of these patches is the terrain model of the WSN deployment. Figure 7 is a terrain model of WSN deployment environment. Suppose in a $100 \mathrm{~m} \times 100 \mathrm{~m} \times 100 \mathrm{~m}$ cube space, we deploy 500 nodes, where the proportion of beacon nodes is $5 \% \sim 30 \%$, communication range is 40 , using Formula (3)for terrain model of the natural shape.

$$
Z=2 e^{-0.008 x^{2}-0.005 y^{2}-2}
$$

For simulation of Figure 7, the experiments are under the MATLAB. First, deploy unknown nodes and beacon nodes, and localization using MSAT3D AOA. Then, use Delaunay triangulation to construct triangle network, as shown in Figure 8. For Terrain Modeling Error (TME), we can use Formula (4), where $z_{i}$ is the real height of node $i$,

$Z_{i}^{\prime}$ is the estimated height.

$$
T M E=\frac{1}{n} \sum_{i=1}^{n}\left|z_{i}-z_{i}^{\prime}\right|
$$

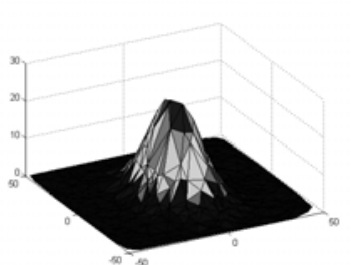

Fig 6. Terrain Model of Deployment

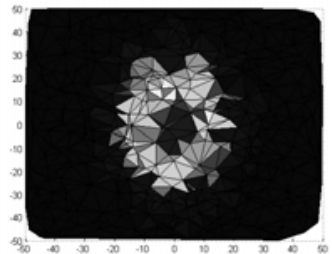

Fig 8 . Delaunay Triangulation of Sensor Nodes

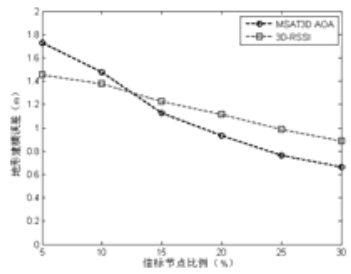

Fig 7. TME Comparison

Figure 9 is the terrain modeling error of MSAT3D AOA and RSSI based 3D localization algorithm under above terrain conditions. We can see that when the beacons number is small, MSAT3D AOA's TME is a slightly larger than RSSI based. But when the ratio of beacons increasing, TME of the proposed algorithm will rapidly decline, which is due to that when the ratio of beacons increase, the multi-hop localization ratio will reduce, and the localization accuracy will be improved.

\section{CONCLUSION}

In this paper, we start from problems of 3D localization, analyzing related research, considering application of outdoor scenes, and on the basis of the simple 3D AOA, with the $3 \mathrm{D}$ angle transmission, proposes a Localization Algorithm of Multi-hop 3D AOA with Space-based Angle Transmission, called MSAT3D. MSAT3D allows that unknown nodes who has less than two beacon nodes, can make use of two neighbors' common neighbor beacons to get beacon information one hop outside, and combines with simple 3D AOA to reduce the number of nodes which can't be located, then it will improve the localization cover ratio. Then using MSAT3D AOA in terrain modeling, further verify the feasibility of the algorithm.

\section{ACKNOWLEDGEMENTS}

I would like to express my sincere appreciation to the Natural science Foundation of Zhejiang Province under (Grant No.LY12F02036, Grant No.LQ12F02015) and Science and Technology Research Project of Zhejiang Province under (Grant No.2011C21014).

\section{REFERENCES}

[1] C.Y.Chong, S.Kumar. Sensor Networks: Evolution, Opportunities, and Challenges. Proc of the IEEE, 2003, 91(8): 1247-1256.

[2] Fan Maojun. Sensor Technology-Neurons of Information Technology Weapons and Equipment. Beijing: National Defence Industry Press, 2008, 154-156.

[3] G.Q. Mao, Baris Fidan, Brian D.O. Anderson. Wireless sensor network localization techniques. Computer Networks, 2007, 51, 2529-2553.

[4] L.Q. Zhang, X.B. Zhou, Q. Cheng. Landscape-3D: A Robust Localization Scheme for Sensor Networks over Complex 3D Terrains. Proc of 2006 IEEE Conf on Local Computer Networks, 2006, 239-246.

[5] Y.H. Liu, J.H. Pu, Y. He, et al. Three-Dimensional Self-Localization Scheme for Wireless Sensor Networks. Journal of Beijing University of Aeronautics and Astronautics, 2008, 34(6): 647-651.

[6] R.H. Huan, Q.Z Chen, K.J. Mao, Y. Pan. A Three-dimension Localization Algorithm for Wireless Sensor Network Nodes Based on SVM. The 1st International Conf on Green Circuits and Systems (ICGCS), 2010, 651-654.

[7] S Roy, S Chatterjee, S Bandyopadhyay. Neighborhood Tracking and Location Estimation of Nodes in Ad Hoc Networks Using Directional Antenna: A Testbed Implementation. IEEE Proc of the Int Conf on Wireless Networks, Communications and Mobile Computing, 2005, 1-6.

[8] D Niculescu, B Nath. DV Based Positioning in Ad Hoc Networks. Journal of Telecommunication Systems, 2003, 22(1/4): 267-280.

[9] D Niculescu, B Nath. Ad hoc positioning system. IEEE GLOBECOM, 2001, 5: 2926-2931.

[10] S Capkun, M Hamdi, Hubaux J P. GPS-Free Positioning in Mobile Ad-hoc Networks. Proc of the 34th Annual Hawaii Int'l Conf on System Sciences, USA: IEEE Computer Society, 2001, 3481-3490.

[11] X.F. Wang, F.Q. Dai, F.Y Miao. Three-Dimensional Wireless Sensor Network Localization Algorithm Based on TOA. Automation and Instrumentation, 2008, 12: 1-9.

[12] L.M. Sun, J.Z. Li, Y. Chen. Wireless Sensor Networks. Beijing: TsingHua University Press, 2008, 140-155.

[13] D Niculescu, B Nath. Ad Hoc Positioning System (APS) Using AOA. Proc 22nd Annual Joint Conf of the IEEE Computer and Communication Societies (INFOCOM 2003), 2003, 3: 1734-1743.

[14] X.B. Wu, S.X Wang, C.S Xiao. A New Study of Delaunay Triangulation Creation. AGEAS, 1999, 28(1): 28-35.

[15] Level Based Path Selection Technique in Large WSN for Hierarchical Architecture.

[16] Comparative Investigations on Performance of Routing Protocols in Presence of Realistic Radio Models for WSNs.

[17] Level Based Path Selection Technique in Large WSN for Hierarchical Architecture.

[18] Energy Efficient Key Management Protocol in Wireless Sensor Networks.

[19] Secure distribution of neural networks in wireless sensor networks 